\title{
Characterization of open cracks in burst vibrothermography experiments
}

\author{
by K. Martínez ${ }^{\star \star \star \star}$, R Celorrio ${ }^{\star \star \star}$, A. Mendioroz*

\begin{abstract}
* Departamento de Física Aplicada I, Escuela de Ingeniería de Bilbao, Universidad del País Vasco UPV/EHU, Plaza Ingeniero Torres Quevedo 1, 48013 Bilbao, Spain, arantza.mendioroz@ehu.eus **Instituto Politécnico Nacional, (IPN), Centro de Investigación en Ciencia Avanzada y Tecnología Avanzada (CICATA), Unidad Legaria, Legaria 694, Col. Irrigación, C. P. 11500, Ciudad de México, Mexico, kmartinez1709@gmail.com

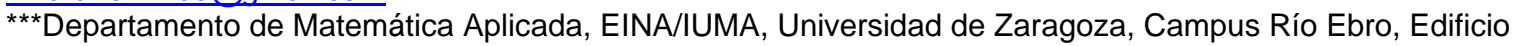
Torres Quevedo, 50018 Zaragoza, Spain, celorrio@unizar.es
\end{abstract}

\begin{abstract}
In this work, we use vibrothermography to characterize open vertical cracks in a fast way. We excite the sample by a brief ultrasound burst and we record the evolution of the surface temperature distribution with an IR camera. From this sequence, temporal and spatial information is selected to characterize the heat sources activated at the defects. This ill-posed inverse problem is solved by inverting the data with a dedicated stabilized inversion algorithm. Experiments performed on samples containing artificial calibrated heat sources confirm the validity of the method to characterize both homogeneous and inhomogeneous flux distributions generated at the defects.
\end{abstract}

\section{Introduction}

Ultrasound excited thermography or vibrothermography [1] is a non-destructive thermographic technique that has been applied very successfully, mainly to detect planar defects with contacting surfaces, such as cracks [2-7], corrosion [8], delaminations [9-13], etc. In this technique, the sample is excited with ultrasounds and at the defects, the relative motion of the two faces produces heat. This thermal energy propagates in the material and the defect is identified as a hot spot at the surface of the specimen with an infrared (IR) video camera. This technique can be applied to any kind of material with the only restriction of not being brittle. In metals, where bulk damping of the ultrasounds is low, it becomes a defectselective technique as heat is only produced at the flaws.

Vibrothermography has been applied not only to detect but also to characterize planar defects [14-17]. In particular, vertical kissing cracks have been characterized using both, modulated [14-16] and burst excitations [17]. It is important to note that, as the information provided by the experiment is thermal (temperature rise at the surface) the physical quantity that can be characterized is the heat flux distribution generated at the crack by the ultrasounds. In kissing cracks, where the two lips are in contact, heat is very likely produced along the whole crack surface [18]. In these conditions, characterizing the area where heat is produced is equivalent to characterizing the crack itself. However, if the crack is open, heat will not be produced along all the crack surface, but just at locations where the two lips are in contact and they move relative to each other [19]. In such a case, the geometry of the heat flux generated at the crack is smaller than the actual crack.

The problem of identifying the heat flux distribution generated at a crack without any previous information of the crack geometry is a severely ill-posed inverse problem [20]. This can be understood due to the diffusive nature of heat propagation: two different heat flux distributions can give rise to very similar temperature distributions at the surface. In the presence of noise, this ill-posedness makes least squares minimization extremely unstable.

In a previous work [17], we developed a stabilized inversion algorithm, to characterize vertical kissing cracks only assuming knowledge of the plane containing the cracks as prior information. The algorithm was successfully applied to size kissing cracks that generate homogeneous and compact heat sources in vibrothermography experiments. In this work, we address more general and realistic situations, such as the excitation of open cracks where heat is not produced all along the crack surface. In the common case of half-penny-shaped open surface breaking cracks, heat production takes place along a certain contour, rather than a compact area [19]. Moreover, the heat flux is very likely non-uniform along this area. Accordingly, as a further step to approach real situations, we also consider non-homogeneous heat source distributions. In section 2 we present the direct surface temperature calculations corresponding to semi-circular stripeshaped heat sources and we introduce the inverse problem. In section 3, we present inversions of synthetic data with added noise generated by semi-circular stripes with different distributions of the heat fluxes. We check the quality of the inversions as a function of the noise level in the data and the particular heat flux distribution. In section 4 we present the set-up, the calibrated samples we have prepared and inversions of experimental data. The results show that it is possible to characterize the heat flux generated by vertical cracks of any shape from burst vibrothermography data, in a fast way. 


\section{Direct and inverse problems}

Our approach to characterize the flux distribution produced in vibrothermography experiments consists in performing a least squares fitting of the data to a semi-analytical model. In this section, we present the calculation of the evolution of the surface temperature distribution produced by vertical heat sources of semi-circular shape. Then, we introduce the inverse problem and our approach to stabilize the inversion.

\subsection{Direct problem}

The first step to characterize the heat source distribution produced in a vibrothermography experiment is to solve the direct problem. We now present the calculation of the evolution of the surface $(z=0)$ temperature distribution produced by a vertical heat source, occupying an area $\Omega$ in plane $\Pi(x=0)$, perpendicular to the sample surface, that emits a constant flux $Q(\vec{r})$ during a time interval $[0, \tau]$. The geometry is depicted in figure $1 \mathrm{a}$.

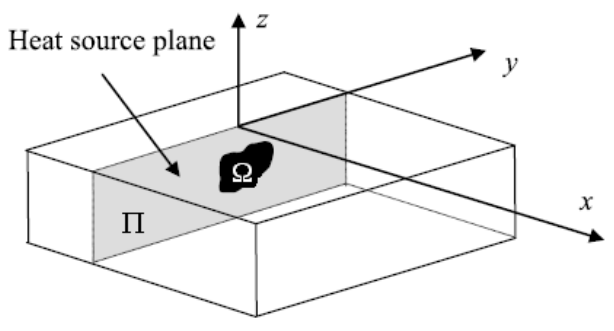

(a)

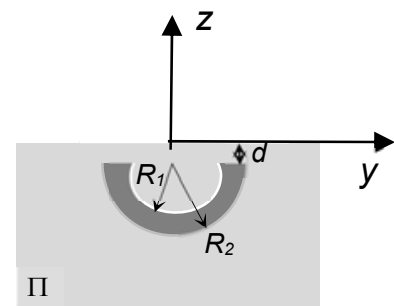

(b)

Fig. 1. a) Geometry of a heat source of area $\Omega$ contained in plane $\Pi(x=0) b)$ Semi-circular stripe-shaped heat source of inner and outer radii $R_{1}$ and $R_{2}$, respectively, buried at a depth $d$.

The calculation can be performed by starting from the temperature produced by a point-like and instantaneous heat source in an infinite medium (Green function) and performing a temporal integration over the duration of the burst $(\tau)$ and a spatial integration over the area covered by the heat sources $\Omega$. Assuming that the sample is semi-infinite, the effect of the surface can be taken into account by assuming adiabatic conditions and applying the images method, i.e., considering the contribution of a reflected image of the heat source at the surface. The resulting expressions for the surface temperature in a medium of thermal conductivity $K$ and diffusivity $D$, during and after the excitation are [17]:

$$
\begin{array}{ll}
T_{h}\left(\vec{r}_{z=0}, t\right)=\iint_{\Omega} \frac{Q\left(\vec{r}^{\prime}\right)}{2 \pi K\left|\vec{r}-\vec{r}^{\prime}\right|} \operatorname{Erfc}\left[\frac{\left|\vec{r}-\vec{r}^{\prime}\right|}{\sqrt{4 D t}}\right] d s^{\prime} & 0 \leq t \leq \tau \\
T_{c}\left(\vec{r}_{z=0}, t\right)=\iint_{\Omega} \frac{Q\left(\vec{r}^{\prime}\right)}{2 \pi K\left|\vec{r}-\vec{r}^{\prime}\right|}\left\{\operatorname{Erfc}\left[\frac{\left|\vec{r}-\vec{r}^{\prime}\right|}{\sqrt{4 D t}}\right]-\operatorname{Erfc}\left[\frac{\left|\vec{r}-\vec{r}^{\prime}\right|}{\sqrt{4 D(t-\tau)}}\right]\right\} d s^{\prime} & \tau<t
\end{array}
$$

In the particular case of the geometry depicted in figure 1b, Eqs. (1a) and (1b) write:

$$
\begin{array}{ll}
T_{h}(x, y, 0, t)=\int_{R_{1}}^{R_{2}} \int_{0}^{\Pi} \frac{Q\left(\vec{r}^{\prime}\right)}{2 \pi K A} E r f c\left[\frac{A}{\sqrt{4 D t}}\right] r^{\prime} d r^{\prime} d \varphi^{\prime} & 0 \leq t \leq \tau \\
T_{c}(x, y, 0, t)=\int_{R_{1}}^{R_{2}} \int_{0}^{\Pi} \frac{Q\left(\vec{r}^{\prime}\right)}{2 \pi K A}\left\{E r f c\left[\frac{A}{\sqrt{4 D t}}\right]-E r f c\left[\frac{A}{\sqrt{4 D(t-\tau)}}\right]\right\} r^{\prime} d r^{\prime} d \varphi^{\prime} & \tau<t
\end{array}
$$

Where $A=\sqrt{\left(y-r^{\prime} \cos \varphi^{\prime}\right)^{2}+\left(d+r^{\prime} \sin \varphi^{\prime}\right)^{2}}$, and $r^{\prime}$ and $\varphi^{\prime}$ are the planar spherical coordinates.

Among all the information contained in Eqs. (2a) and (2b), which describe the complete evolution of the surface temperature distribution, in order to characterize the heat flux generated at the crack we select spatial and temporal information. This data reduction makes the inversion algorithm fast, which is important for practical applications. The information we select is the thermogram obtained at the end of the burst $T_{r}=T(\mathrm{x}, \mathrm{y}, 0, \tau)$, and the evolution of the temperature at the central pixel, $T_{t}=T(0,0,0, t)$, combined in $T=\left(T_{r}, T_{t}\right)^{T}$, where superscript $T$ stands for transpose. In section 3 we will generate synthetic $T_{r}$ and $T_{t}$ data from Eqs. (2a) and (2b), which will be introduced in the inversion algorithm. 


\subsection{Inverse problem}

The inverse problem we address consists in retrieving the heat flux distribution responsible for the surface temperature that we measure in an experiment. The experimental data $T^{\delta}=\left(T_{r}^{\delta}, T_{t}^{\delta}\right)^{T}$ are affected by a certain noise level $\delta$ that we define as:

$$
\delta^{2}=\left\|T^{\delta}-T\right\|^{2}
$$

The geometry of the heat source being unknown, the inversion cannot be addressed as a parameter estimation problem. The procedure we follow consists in meshing plane $\Pi(x=0)$ and finding the heat source at each node, $Q^{\delta}$, that minimizes the residual $R^{2}$, i. e., the norm of the squared differences between the experimental and calculated surface temperature data:

$$
R^{2}:=\left\|T_{\text {calc }}\left(Q^{\delta}\right)-T^{\delta}\right\|^{2}
$$

This involves determining a very large number of unknowns. Under these circumstances, the inverse problem is severely ill-posed, and the minimization of the residual in Eq. (4) is unstable. The strategy we apply to stabilize the inversion is to add some penalty terms to the right-hand side of Eq. (4), based on Tikhonov (TKo) [21], Total Variation (TV) [22], and Lasso $\left(L_{1}\right)$ functionals [23]:

$$
\begin{aligned}
& T K_{0}(Q)=\iint_{\Pi}|Q|^{2} d s \\
& T V(Q)=\iint_{\Pi}|\nabla Q| d s \\
& L_{1}(Q)=\iint_{\Pi}|Q| d s
\end{aligned}
$$

The choice of these functionals is made according to the characteristics of the flux distribution we want to retrieve. $T V$ searches for functions with flat sections and sharp edges, like the confined flux distributions we seek, and $L_{1}$ reduces the area were the function has non-zero values, which enhances the contrast [24]. TKo is introduced just to start the minimization. The drawback with both $T V$ and $L_{1}$ is that neither is a quadratic, even differentiable operator, so we make use of quadratic functionals to approximate both penalty terms in an iterative way, using lagged-diffusivity fixed-point iterations [25] that include a small positive parameter $\varepsilon$ that is introduced in order to avoid dividing by zero:

$$
\begin{aligned}
& T V(Q)=\iint_{\Pi}|\nabla Q| d s \simeq \lim _{j \rightarrow \infty} \iint_{\Pi} \frac{\left|\nabla Q_{j}\right|^{2}}{\sqrt{\varepsilon+\left|\nabla Q_{j-1}\right|^{2}}} d s=\lim _{j \rightarrow \infty} \iint_{\Pi} \frac{\left(\partial_{y} Q_{j}\right)^{2}+\left(\partial_{z} Q_{j}\right)^{2}}{\sqrt{\varepsilon+\left(\partial_{y} Q_{j-1}\right)^{2}+\left(\partial_{z} Q_{j-1}\right)^{2}}} d s \\
& L_{1}(Q)=\iint_{\Pi}|Q| d s \simeq \lim _{j \rightarrow \infty} \iint_{\Pi} \frac{\left|Q_{j}\right|^{2}}{\sqrt{\varepsilon+\left|Q_{j-1}\right|^{2}}} d s
\end{aligned}
$$

The new residual to be minimized is:

$$
R_{\vec{\alpha}^{i}}^{2}=\alpha_{T K}^{i} T K_{0}\left(Q^{\delta, \vec{\alpha}^{i}}\right)+\alpha_{T V}^{i} T V\left(Q^{\delta, \vec{\alpha}^{i}}\right)+\alpha^{i} L_{1}\left(Q^{\delta, \vec{\alpha}^{i}}\right)+\left\|T_{\text {calc }}\left(Q^{\delta,,^{i}}\right)-T^{\delta}\right\|^{2}
$$

Each penalty term in Eq. 8 is multiplied by a regularization parameter $\left(\alpha_{T K}^{i}, \alpha_{T V}^{i}, \alpha_{L_{1}}^{i}\right)$, combined in $\vec{\alpha}^{i}=\left(\alpha_{T K}^{i}, \alpha_{T V}^{i}, \alpha_{L_{1}}^{i}\right)$. These three parameters determine the size of the penalty terms with respect to the last term in the right-hand side of Eq. 8, called the discrepancy term, which represents the quantity we want to minimize. High values of the regularization parameters stabilize the inversion but introduce an error in the solution, $Q^{\delta, \bar{\alpha}^{i}}$. In order to find a trade-off between stability of the inversion and accuracy of the solution, we reduce the regularization parameters in successive iterations $i$, and we stop the iterations when the discrepancy term reaches the noise level in the data.

\section{Inversion of synthetic data}

We have first tested the inversion algorithm by inverting synthetic $T_{r}$ and $T_{t}$ data, calculated from Equations (2a) and (2b) with homogeneous flux and material properties corresponding to AISI-304 stainless steel, the material our samples are made of: $D=4 \mathrm{~mm}^{2} / \mathrm{s}, K=15 \mathrm{~W} / \mathrm{mK}$. Then, we add random noise to the data, representing the desired noise level. We first start with homogeneous distributions. As an example, in figure 2 we show $T_{r}$ and $T_{t}$ calculated for a heat source of inner and outer radii $R_{1}=1 \mathrm{~mm}$ and $R_{2}=2 \mathrm{~mm}$, respectively, buried a depth of $d=100 \mathrm{~mm}$, and applying a burst of $\tau=2 \mathrm{~s}$, with $5 \%$ added noise. 


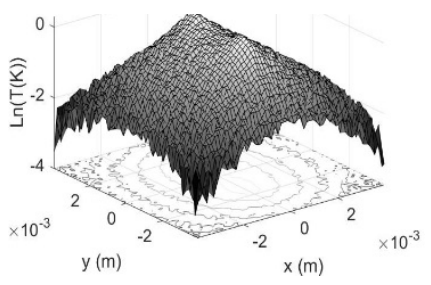

(a)

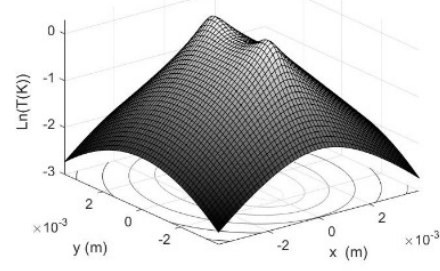

(b)

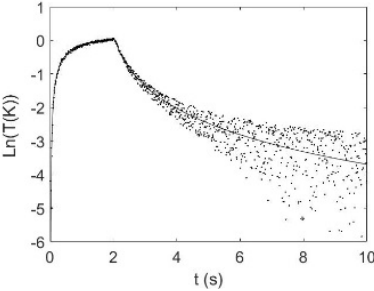

(c)

Fig. 2. (a) Noisy (5\%) $T_{r}$ corresponding to a semi-circular stripe-shaped and homogeneous heat source of radii $R_{1}=1$ $\mathrm{mm}$ and $R_{2}=2 \mathrm{~mm}$, a depth of $d=100 \mu \mathrm{m}$ and a burst of $\tau=2 \mathrm{~s}$. (b) Fitted thermogram, and (c) noisy (symbols) and fitted (continuous line) $T_{t}$.

The retrieved heat flux distribution is depicted in a grey level diagram in figure $3 a$ (middle): white represents maximum flux and black is absence of heat sources. The real contour of the homogeneous heat source is depicted in red. As can be seen, the quality of the reconstruction is very good. We have also generated data for the same flux distribution, but for other burst durations, keeping an added noise of $5 \%$. The results are depicted in figure $3 a$. As can be seen, the results do not significantly depend on the burst duration. However, if we reduce the thickness of the semi-circular stripe, the effect of the burst duration is more pronounced. As can be seen in figure $3 \mathrm{~b}$, short bursts provide better reconstructions of a semi-circular stripe $0.2 \mathrm{~mm}$ thick $\left(R_{1}=1 \mathrm{~mm}\right.$ and $\left.R_{2}=1.2 \mathrm{~mm}\right)$. The high frequency content in the short burst being relatively larger than in the long one, the former is able to provide better identification of thin features

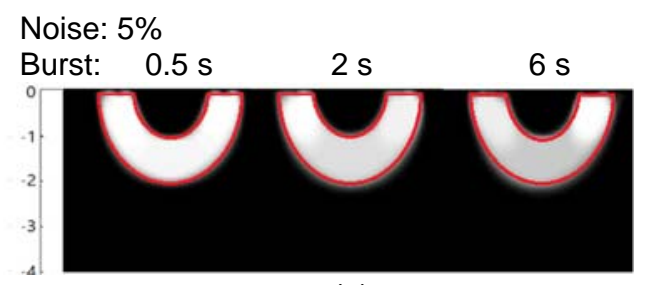

$z(\mathrm{~mm})$

(a)

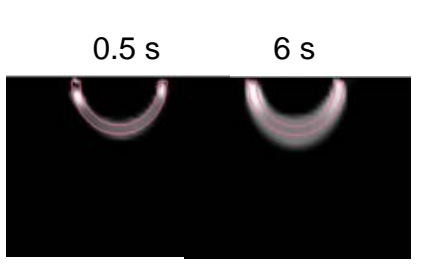

(b)
Burst: $5 \mathrm{~s}$

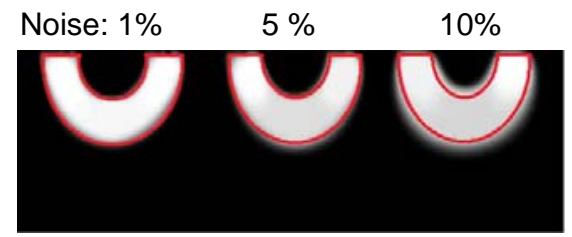

(c)

Fig. 3. Grey level representation of the heat flux distribution retrieved in plane $\Pi$ : white is maximum flux and black is absence of heat sources. The real contour of the heat source distribution depicted in red. (a) Reconstructions obtained for a noise level of $5 \%$ and burst durations $\tau=0.5,2$ and $6 \mathrm{~s}$, for a semi-circular stripe of inner and outer radii $R_{1}=1 \mathrm{~mm}$ and $R_{2}=2 \mathrm{~mm}$, respectively. (b) Same as (a) but for $\tau=0.5$ and $6 \mathrm{~s}$ and $R_{1}=1 \mathrm{~mm}$ and $R_{2}=1.2 \mathrm{~mm}$ (b) Reconstructions obtained for a burst $\tau=5 \mathrm{~s}$ and noise levels of 1,5 , and $10 \%$.

We have also checked the robustness of the method to the noise level in the data. In figure $3 \mathrm{c}$, we show reconstructions obtained for the same homogeneous heat flux distribution as in figure $3 \mathrm{a}$, and a burst duration of $\tau=5 \mathrm{~s}$, but with different noise levels: 1,5 and $10 \%$. Although the accuracy of the reconstructions is good, they show a progressive degradation as the noise level increases: the inversion of data with $10 \%$ noise clearly overflows the real contour.

As a further step to approach situations with real cracks, we have also considered position-dependent fluxes within the red contours depicted in figure 3. We have analysed the cases of depth-, radius-, and angle-dependent heat fluxes. In figure 4 we show the reconstructions obtained from $T_{r}$ and $T_{t}$ data obtained for a burst of $\tau=2 \mathrm{~s}$ and three different flux distributions within a semi-circular stripe of inner radius $R_{1}=1 \mathrm{~mm}$, outer radius $R_{2}=2 \mathrm{~mm}$ and a depth of $d=0.1 \mathrm{~mm}$. In each figure, the reconstruction on the left correspond to data affected by $1 \%$ noise, and the one on the right to $5 \%$. The real flux distributions are displayed at the bottom.

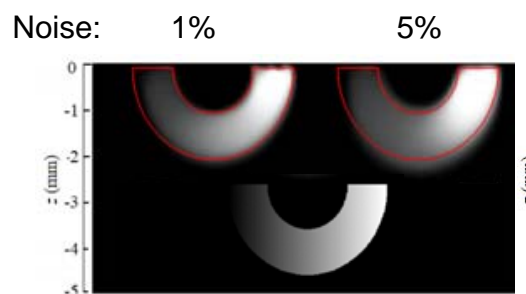

(a)
$1 \%$

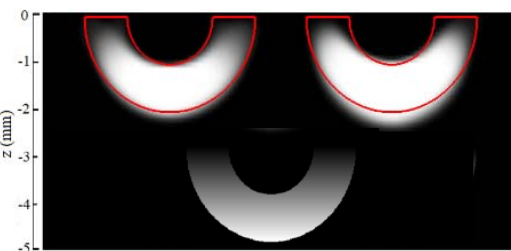

(b)
$1 \%$

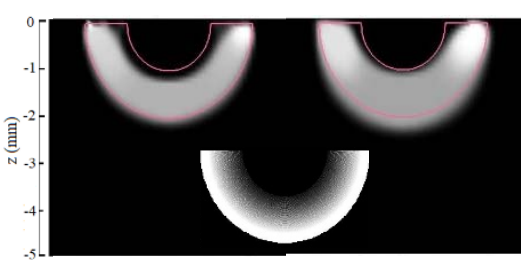

(c)

Fig. 4. Reconstructions corresponding to inhomogeneous fluxes within a semi-circular stripe of inner and outer radii $R_{1}=$ $1 \mathrm{~mm}$ and $R_{2}=2 \mathrm{~mm}$, respectively, and a depth of $d=0.1 \mathrm{~mm}$, obtained from temperature data calculated for a burst of $\tau$

$=2 s$, and different noise levels. (a) Angle-dependent flux, (b) depth-dependent flux and (c) radius-dependent flux.

Reconstructions on top and real flux distribution at the bottom. 
The reconstructions corresponding to an angle-dependent flux (figure 4a) are in excellent agreement with the real flux for noise level of $1 \%$ and $5 \%$. Regarding the depth-dependent fluxes, the results in figure $4 \mathrm{~b}$ indicate that for a $1 \%$ noise level the reconstruction is good, but the accuracy decreases as the noise level increases, so the noise level should be kept as low as possible to accurately retrieve depth-dependent heat fluxes. Finally, the results in figure 4c indicate that the method cannot retrieve radius-dependent fluxes.

As we mentioned in section 2.2, despite the fact that introducing a TV term in the residual drives the search of the solution among blocky functions, the results depicted in figure 4 point out that, not only homogeneous but also inhomogeneous heat sources can be identified. The results improve the smoother the variation of the heat flux distribution. A plausible reason for this is that Eqs. 6 and 7 represent a smooth approximation of $T V$ and $L_{1}$ functionals. Accordingly, the method works for both homogeneous and inhomogeneous distributions: the solutions are blocky functions when the flux is homogeneous within a delimited area (see figure 3), and in the case of inhomogeneity, the retrieved flux distribution is very satisfactorily identified when the variation is smooth.

Finally, in order to compare the predictions from synthetic data with experimental results, we present reconstructions obtained from data calculated for a semi-circular stripe-shaped heat source, divided into two halves, each with homogeneous (but different) heat fluxes: the flux in one half is double than in the other. The results for burst durations of $\tau=0.5 \mathrm{~s}$ and $4 \mathrm{~s}$ are depicted in figure 5 . It shows that it is possible to identify the two halves in the reconstruction and that the values we obtain for the heat flux on the left hand side of each semi-circle is double the radius we obtain on the right hand side. All these results point out that the method has the potential to quantify inhomogeneous heat fluxes excited in vibrothermography experiments in a fast way: the inversions presented in this section take about $15 \mathrm{~s}$ in a PC.

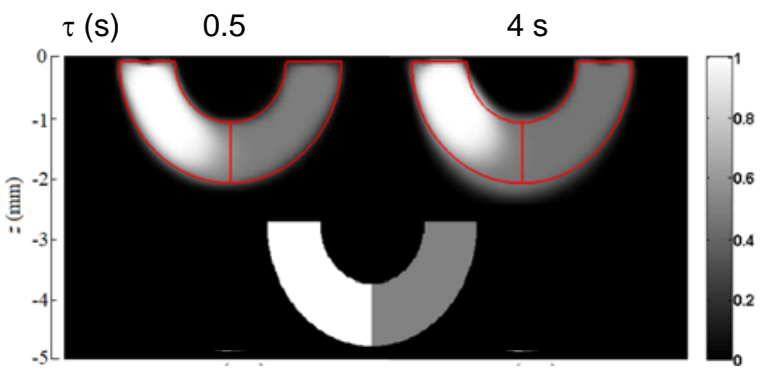

Fig. 5. Reconstructions (top) obtained for a stripe-shaped heat source $\left(R_{1}=1 \mathrm{~mm}, R_{2}=2 \mathrm{~mm} \mathrm{~d}=0.1 \mathrm{~mm}\right)$, divided into two halves (2:1 flux ratio), with bursts durations of $\tau=0.5$ and $4 \mathrm{~s}$ and data affected with $5 \%$ noise. The real flux is displayed at the bottom.

\section{Experiments}

In order to check the ability of the method to characterize heat sources generated by open surface breaking vertical cracks in vibrothermography experiments, we have prepared samples containing calibrated stripe-shaped heat sources when excited with ultrasounds. The samples consist of two identical AISI-304 stainless steel parts, each with a flat, well rectified surface that matches the counterpart. We put a $38 \mu \mathrm{m}$ thick $\mathrm{Cu}$ tape of the desired geometry and dimensions between the two parts, at a calibrated depth from the surface where we take data. In addition, we put two more $\mathrm{Cu}$ rectangles of the same thickness deep below this surface. The diagram of an open sample is depicted in figure $6 \mathrm{a}$. Then, we attach the two parts with screws (figure 6b). When we launch the ultrasounds (figure 6c), the Cu slabs produce heat due to friction with the steel surfaces. With the two flat surfaces well rectified, the steel parts are parallel to each other so we get homogeneous and calibrated heat sources corresponding to the geometry of the shallow Cu slab.

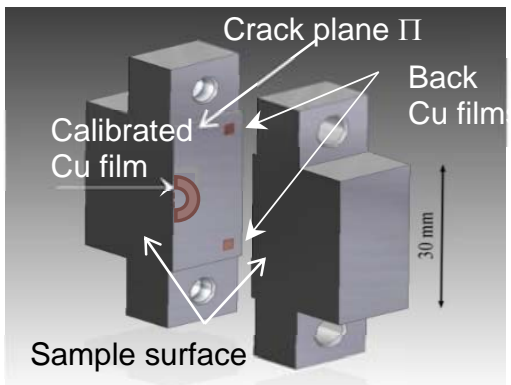

(a)

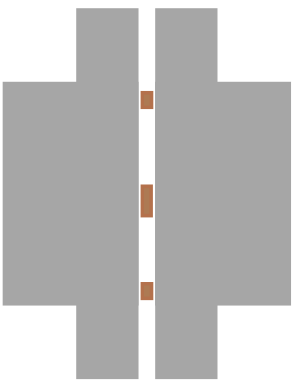

(b)

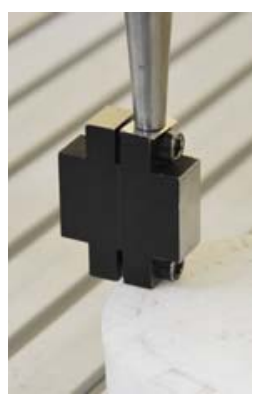

(c)

Fig. 6. Calibrated samples. (a) Diagram of two parts composing the sample, with the calibrated Cu slab in between. (b) Front view (as viewed by the camera) of the sample closed. (c) Picture of the sample closed and in place for excitation. 
We excite the sample with a UTVis equipment from Edevis. The ultrasound frequency is tunable between 15 and $25 \mathrm{kHz}$, with a maximum power of $2.2 \mathrm{~kW}$ at $20 \mathrm{kHz}$. We work at an ultrasound frequency of $23 \mathrm{kHz}$, at which we find the optimum signal in our samples, with ultrasound powers ranging between 200 and $450 \mathrm{~W}$. We use a thin Al film as coupling material between the sonotrode and the sample. We cover the sample surface with black paint in order to improve the emissivity. We collect the radiation coming from the sample with an infrared video camera (JADE J550M from Cedip), sensitive in the 3-5 $\mu \mathrm{m}$ range. The camera is equipped with a $320 \times 240 \mathrm{InSb}$ detector, a $50 \mathrm{~mm}$ focal length lens, and the NETD is $25 \mathrm{mK}$. At the minimum working distance, each pixel in the camera averages the radiation coming from a $135 \mu \mathrm{m}$ side square in the sample.

As an example, in figure 7 we show experimental and fitted $T_{r}$ and $T_{t}$ obtained with a semi-circular Cu stripe with inner radius $R_{1}=0.9 \mathrm{~mm}$, outer radius $R_{2}=2.1 \mathrm{~mm}$ located at a depth $d=0.15 \mathrm{~mm}$, and a burst duration of $\tau=0.5 \mathrm{~s}$.

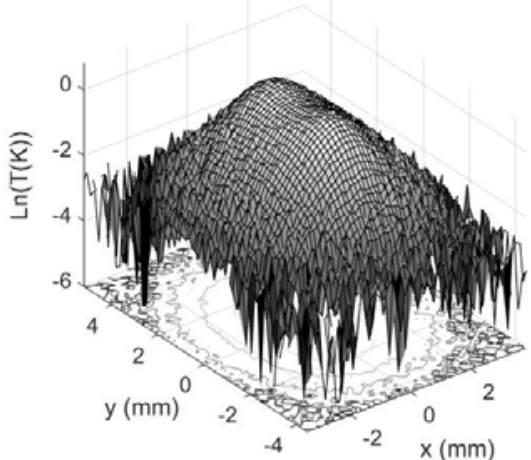

(a)

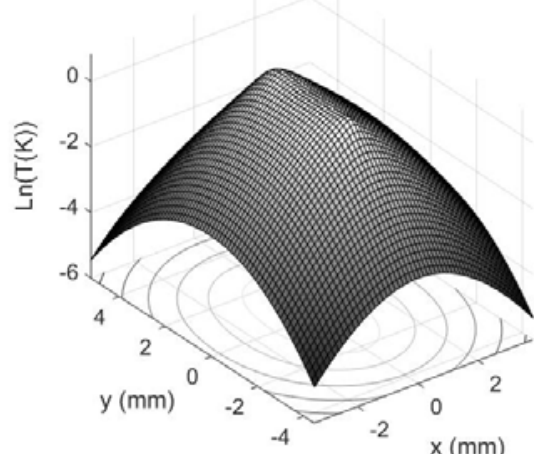

(b)

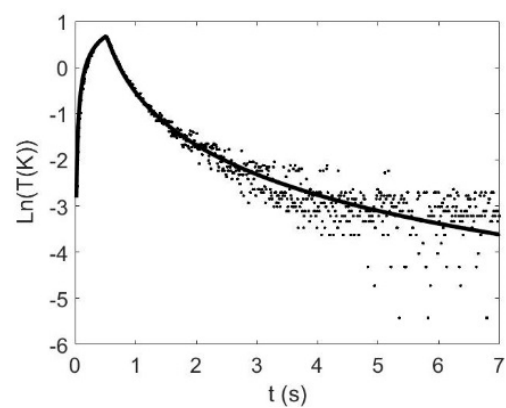

(c)

Fig. 7. (a) Experimental $T_{r}$ corresponding to a semi-circular homogeneous $C u$ stripe of radii $R_{1}=0.9 \mathrm{~mm}$ and $R_{2}=2.1$ $\mathrm{mm}$, and a depth of $d=150 \mu \mathrm{m}$ obtained with a $\tau=0.5 \mathrm{~s}$ burst. (b) fitted thermogram and (c) noisy (symbols) and fitted (continuous line) $T_{t}$.

The reconstruction of these data is depicted in figure 8 (a). We also took data with other durations of the excitation, namely $\tau=1,2$, and $3.5 \mathrm{~s}$. The results are displayed in figures $8 \mathrm{~b}, 8 \mathrm{c}$, and $8 \mathrm{~d}$, respectively. As can be seen, the results are in quite good agreement with the real shape of the Cu stripe.

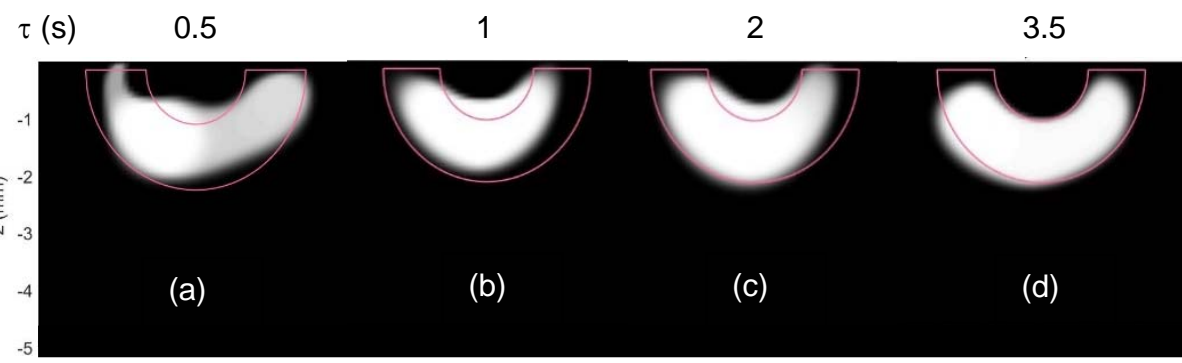

Fig. 8. Grey level representation of the heat flux distribution obtained for a semi-circular Cu stripe of inner and outer radii $R_{1}=0.9 \mathrm{~mm}$ and $R_{2}=2.1 \mathrm{~mm}$, respectively, buried at a depth of $d=0.15 \mathrm{~mm}$ burst durations of $\tau=0.5,1,2$, and $3 \mathrm{~s}$, in (a), (b), (c), and (d), respectively.

We also took data with narrow semi-circular Cu stripes $\left(R_{1}=0.9 \mathrm{~mm}, R_{2}=1.1 \mathrm{~mm}, d=0.1 \mathrm{~mm}, \tau=0.5 \mathrm{~s}\right)$, semicircular stripes buried deeper below the surface $\left(R_{1}=1.1 \mathrm{~mm}, R_{2}=2.2 \mathrm{~mm}, d=1 \mathrm{~mm}, \tau=1.5 \mathrm{~s}\right)$, and with stripes of triangular shape $(\tau=2 \mathrm{~s})$. Figures $9 \mathrm{a}, 9 \mathrm{~b}$, and $9 \mathrm{c}$ show the results in the three cases (top), together with micrographs of the Cu stripes used to take the data (bottom). In the case of the narrow stripe (figure 9a), the Cu "slab" was actually a Cu wire, with a diameter of $150 \mu \mathrm{m}$, that was squashed between the steel parts to get it flattened. The reconstructions are in qualitative good agreement with the real Cu slabs geometry, and feature rounded contours, due to the effect of the TVbased penalty term. This functional penalizes the spatial derivative of the sought function and thus tends to round the contour of the retrieved heat source (where the derivative is large), as a circle represents the shape of shorter contour for a given area. This is the reason why the reconstructions of semi-circles are nicely reproduced, whereas the reconstruction of the triangle (figure $9 \mathrm{c}$ ) is obviously rounded. 
$\tau(\mathrm{s})$
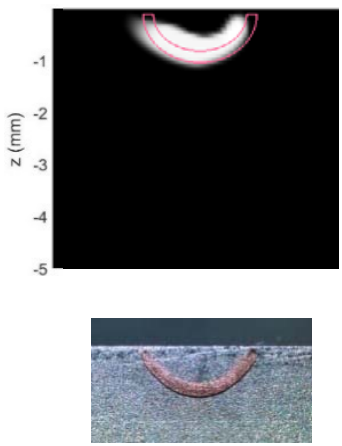

(a)
1.5

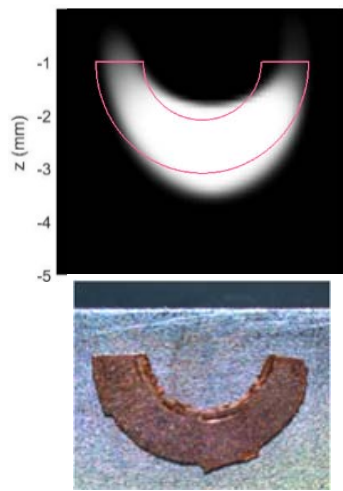

(b)
2

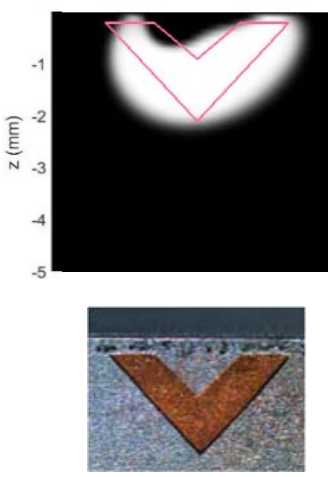

(c)

Fig. 9. Experimental reconstructions (top) and micrograph of the corresponding $\mathrm{Cu}$ stripe (bottom), obtained for (a) a thin semicircular stripe of radii $R_{1}=0.9 \mathrm{~mm}, R_{2}=1.1 \mathrm{~mm}$, buried at a depth $d=0.1 \mathrm{~mm}$ with a $\tau=0.5 \mathrm{~s}$ burst, (b) a semicircular stripe of radii $R_{1}=1.1 \mathrm{~mm}, R_{2}=2.2 \mathrm{~mm}$, buried at a depth $d=1 \mathrm{~mm}$ with a $\tau=1.5 \mathrm{~s}$ burst, and (c) a triangular slab (depth of shallow and deep apices $0.9 \mathrm{~mm}$ and $2.1 \mathrm{~mm}$, respectively, $d=0.2 \mathrm{~mm}$ ) excited by a $\tau=2 \mathrm{~s}$ burst.

As mentioned at the beginning of this section, the design of the calibrated samples with the two parallel flat steel surfaces sandwiching the $\mathrm{Cu}$ slabs, is addressed to produce homogeneous heat sources. Besides, it is certainly challenging to build samples with inhomogeneous and calibrated heat sources activated by the ultrasounds. In order to check the performance of the method with experimental data produced by inhomogeneous heat sources, we have sandwiched two stripes of different materials (annealed and hard $\mathrm{Cu}$ ) each with the approximate shape of a quarter of a circle $\left(R_{1}=0.7 \mathrm{~mm}, R_{2}=1.7 \mathrm{~mm}, d=0.05 \mathrm{~mm}\right)$, forming an approximate full semi-circle when placed in contact on the lower side. The two materials produce different but homogeneous heat fluxes when rubbing with the steel surfaces (in the manner of the synthetic fluxes depicted at the bottom of figure 5). However, we do not have a prior quantitative estimation of the ratio of the fluxes. The reconstruction from data obtained with a $\tau=2 \mathrm{~s}$ burst is depicted in figure 10a, and the micrograph of the slabs used to take the data is shown in figure $10 \mathrm{~b}$.

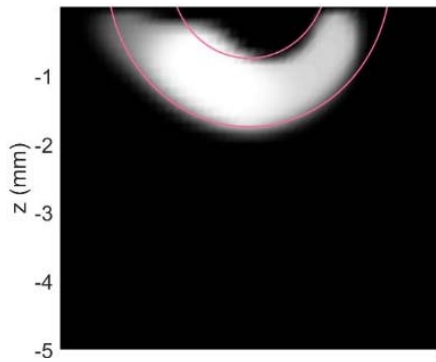

(a)

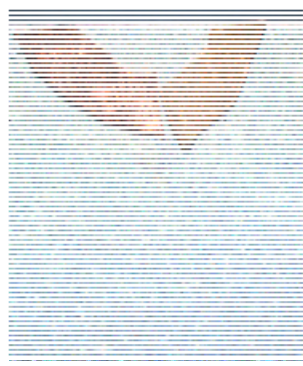

(b)

Fig. 10. (a) Experimental reconstructions obtained with a $\tau=2 \mathrm{~s}$ burst and (b) micrograph of the two Cu types stripes of radii $R_{1}=0.7 \mathrm{~mm}, R_{2}=1.7 \mathrm{~mm}$, buried at a depth of $d=0.05 \mathrm{~mm}$.

The reconstruction shows two different regions (left and right) with different but quasi-homogeneous fluxes, featuring a ratio of 1:0.83. Although we cannot corroborate this flux ratio with independent estimations, the similarity of the fluxes seems sensible as both slabs are made of the same material but different treatment and slightly surface finish.

\section{Summary and conclusions}

In this work, we have addressed the characterization of open, surface breaking vertical cracks using vibrothermography experiments in the burst regime. The specificity of open cracks, if compared with kissing cracks in vibrothermography experiments consists in heat not being produced all along the crack surface, but along the path where the two lips are in contact and move relative to each other. This produces characteristic stripe-shaped (as opposed to compact) heat sources when exciting open surface half-penny cracks. We propose a least square fitting of spatial and temporal information to characterize this heat flux: the thermogram obtained at the end of the burst and the evolution of the temperature at the central pixel. The method involves a search of heat sources along a vertical plane, without any 
assumption on the geometry of the excited heat flux. We have solved this severely ill-posed inverse problem by stabilizing the minimization with penalty terms based on $T V$ and $L_{1}$ functionals. The results of both, synthetic data with added uniform noise and experimental data obtained from samples with calibrated heat sources, indicate that the method is able to characterize accurately homogeneous heat sources activated in vibrothermography experiments with open cracks. Moreover, in the case of inhomogeneous distribution of the flux, we have found that the flux distribution can be identified in the case of smoothly varying fluxes. For an accurate identification of these inhomogeneous fluxes, a high signal-to-noise ratio is ideal. These results open the possibility of estimating the absolute flux distribution generated in vibrothermography experiments, from well calibrated surface temperature data. This information would be extremely valuable to understand the relationship between strain and heat production in experiments with real cracked specimens and to improve excitation conditions.

\section{Acknowledgments}

The authors would like to acknowledge financial support by Ministerio de Economía y Competitividad (DPI201677719-R, AEI/FEDER, UE), by Universidad del País Vasco UPV/EHU (GIU16/33), SIP-IPN (Research Grants 20160144, 20170545) and CONACyT (2015-02-1042, 264093, 2016-01-2482). The support of COFAA-IPN by the SIBE and BEIFI programs is also acknowledged.

\section{REFERENCES}

[1] Henneke E. G.. Jones T. S., Detection of Damage in Composite Materials by vibrothermography, Nondestructive Evaluation and Flaw Criticality for Composite Materials by Amer. Society for Testing and Materials, STP; $696: 99$. 83-95. 1979.

[2] Morbidini M., Cawley P., Barden T., Almond D.. Duffour P., Prediction of the thermosonic signal from fatigue cracks in metals using vibration damping measurements, J. Appl. Phys. - Vol. 100, 104905 (13pp), 2006.

[3] Holland S. D., Uhl C., Ouyang Z., Bantel T., Li M., Meeker W. Q., Lively J., Brasche L., Eisenmann D., Quantifying the vibrothermographic effect, NDT\&E Int. - Vol. 44, pp. 775-782, 2011.

[4] Montanini R., Freni F., Rossi G. L., Quantitative evaluation of hidden defects in cast iron components using ultrasound activated lock-in vibrothermography, Rev. Sci. Instr. - Vol. 83, 094902 (8pp), 2012.

[5] Guo X., Vavilov V., Crack detection in aluminum parts by using ultrasound excited infrared thermography, Infr. Phys. Tech. - Vol. 61, pp. 149-156, 2013.

[6] Piau J. P., Bendada A., Maldague X., Legoux J.-G., Nondestructive testing of open microscopic cracks in plasmasprayed-coatings using ultrasound excited thermography, Nondestr. Test. Eval. - Vol. 23, pp. 109-120, 2008.

[7] Han X., Zeng Z., Li W., Islam M. S., Lu J., Loggins V., Yitamben E., Favro L. D., Newaz G., Thomas R. L., Acoustic chaos for enhanced detectability of cracks by sonic infrared imaging, J. Appl. Phys. - Vol. 95, pp. 3792-3797, 2004.

[8] Salerno A., Dillenz A., Wu D., Rantala J., Busse G., "Progress in ultrasound excited thermography", Proceedings of the 4th Conference on Quantitative IR Thermography, pp. 154-160, 1998.

[9] Pye J., Adams R. D., Heat emission from damaged composite materials and its use in nondestructive testing, J. Phys. D - Vol. 14, pp. 927-941, 1981.

[10] Rantala J., Wu D., Busse G., Amplitude-modulated lock-in vibrothermography for NDE of polymers and composites, Res. Nondestr. Eval. - Vol. 7, pp. 215-228, 1995.

[11] Favro L. D., Han X., Ouyang Z., Sun G., Thomas R. L., Sonic IR imaging of cracks and delaminations, Analitical Sciences. - Vol. 17, pp. 451-453, 2001.

[12] Han X., Favro L. D., Thomas R. L., Sonic IR imaging of delaminations and disbonds in composites, J. Phys D: Appl. Phys. - Vol. 44, 034013 (5pp), 2011.

[13] Barden T. J., Almod D. P., Pickering S. G., Morbidini M., Cawley P., Detection of impact damage in CFRP composites by thermosonics, Nondestr. Test. Eval. - Vol. 22, pp. 71-82, 2007.

[14] Celorrio R., Mendioroz A., Salazar A., Characterization of vertical buried defects using lock-in vibrothermography: II. Inverse problem, Meas. Sci. Technol. - Vol. 24, 065602 (9pp), 2013.

[15] Mendioroz A., Castelo A., Celorrio R., Salazar A., Characterization and spatial resolution of cracks using lock-in vibrothermography, NDT\&E Int. - Vol. 66, pp. 8-15, 2014.

[16] Castelo A., Mendioroz A., Celorrio R., Salazar A., Optimizing the inversion protocol to determine the geometry of vertical cracks from lock-in vibrothermography, J. Nondestr. Eval. - Vol. 36, 3 (12pp), 2017.

[17] Mendioroz A., Celorrio R., Cifuentes A., Zaton L., Salazar A., Sizing vertical cracks using burst vibrothermography, NDT\&E Int. - Vol. 84, pp. 36-46, 2016.

[18] Rothenfusser M., Homma C., "Acoustic thermography: vibrational modes of cracks and the mechanism of heat generation", AIP Conf. Proc. - Vol. 760, pp. 624-631, 2005. 
[19] Renshaw J., Holland S. D., Thomson R. B., Measurement of crack opening stresses and crack closure stress profiles from heat generation in vibrating cracks, Appl. Phys. Lett. - Vol. 93, 081914 (3pp), 2008.

[20] Wahba G., IIl-posed problems: numerical and statistical methods for mildly, moderately and severely ill-posed problems with noisy data, Technical Report no 595 (Madison, University of Wisconsin), 1980.

[21] Engl H.W., Hanke M., Neubauer A., Regularization of Inverse Problems, Kluwer Academic Publisher, Dordrecht, 2000.

[22] Brune C., Sawatzky A., Burger M., Primal and Dual Bregman Methods with Application to Optical Nanoscopy, Int. J. Comput. Vis. - Vol. 92, pp. 211-229, 2011.

[23] Daubechies I., Defrise M., De Mol C. A., An iterative thresholding algorithm for linear inverse problems with a sparsity constraint., Commun.. Pure Appl. Math. -Vol. 11, pp. 1413-1457, 2004.

[24] Tibshirani R., Regression shrinkage and selection via the lasso, J, R Stat. Soc. Ser. B (Method.). -Vol. 58, pp. 267-288, 1996.

[25] Vogel C.R., Computational methods for inverse problems. Philadelphia:SIAM; 2002. 